\title{
Molecular Subtype Classification in Lower-Grade Glioma with Accelerated DTI
}

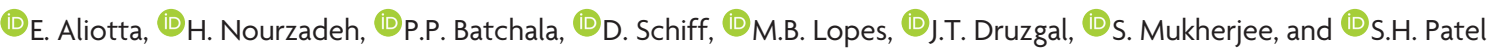

\begin{abstract}
BACKGROUND AND PURPOSE: Image-based classification of lower-grade glioma molecular subtypes has substantial prognostic value. Diffusion tensor imaging has shown promise in lower-grade glioma subtyping but currently requires lengthy, nonstandard acquisitions. Our goal was to investigate lower-grade glioma classification using a machine learning technique that estimates fractional anisotropy from accelerated diffusion MR imaging scans containing only 3 diffusion-encoding directions.
\end{abstract}

MATERIALS AND METHODS: Patients with lower-grade gliomas $(n=41)$ (World Health Organization grades II and III) with known isocitrate dehydrogenase (IDH) mutation and 1p/19q codeletion status were imaged preoperatively with DTI. Whole-tumor volumes were autodelineated using conventional anatomic MR imaging sequences. In addition to conventional ADC and fractional anisotropy reconstructions, fractional anisotropy estimates were computed from 3-direction DTI subsets using DiffNet, a neural network that directly computes fractional anisotropy from raw DTI data. Differences in whole-tumor ADC, fractional anisotropy, and estimated fractional anisotropy were assessed between IDH-wild-type and IDH-mutant lower-grade gliomas with and without 1p/19q codeletion. Multivariate classification models were developed using whole-tumor histogram and texture features from ADC, ADC + fractional anisotropy, and $A D C+$ estimated fractional anisotropy to identify the added value provided by fractional anisotropy and estimated fractional anisotropy.

RESULTS: $A D C(P=.008)$, fractional anisotropy $(P<.001)$, and estimated fractional anisotropy $(P<.001)$ significantly differed between $I D H$-wild-type and IDH-mutant lower-grade gliomas. ADC $(P<.001)$ significantly differed between IDH-mutant gliomas with and without codeletion. ADC-only multivariate classification predicted IDH mutation status with an area under the curve of 0.81 and codeletion status with an area under the curve of 0.83. Performance improved to area under the curve $=0.90 / 0.94$ for the ADC + fractional anisotropy classification and to area under the curve $=0.89 / 0.89$ for the $A D C+$ estimated fractional anisotropy classification.

CONCLUSIONS: Fractional anisotropy estimates made from accelerated 3-direction DTI scans add value in classifying lower-grade glioma molecular status.

ABBREVIATIONS: $\mathrm{AUC}=$ area under the curve; Codel $=$ codeletion; $\mathrm{dFA}=$ fractional anisotropy estimates; $\mathrm{FA}=$ fractional anisotropy; $\mathrm{LGG}=$ lower-grade glioma; MUT = mutated; Noncodel = noncodeleted; $W T=$ wild-type; $I D H=$ isocitrate dehydrogenase

$\mathrm{T}$ he classification of diffuse lower-grade gliomas (LGGs) into molecular subtypes as designated by the World Health Organization in 2016 has important prognostic implications. Median survival in LGG is $>6$ years in the presence of an isocitrate dehy-

Received April 16, 2019; accepted after revision July 1.

From the Departments of Radiation Oncology (E.A., H.N.), Radiology (P.P.B., J.T.D., S.M., S.H.P.), Neurology (D.S.), and Pathology (Neuropathology) (M.B.L.), University of Virginia, Charlottesville, Virginia.

Sohil H. Patel was supported by a Radiological Society of North America Research Scholar Grant (RSCH1819).

Please address correspondence to Eric Aliotta, PhD, Radiological Physics, University of Virginia, 1335 Lee St, Box 800375, Charlottesville, VA 22908; e-mail: eric.aliotta@virginia.edu

三 Indicates article with supplemental on-line tables.

http://dx.doi.org/10.3174/ajnr.A6162 drogenase $(I D H)$ gene mutation $\left(I D H_{\mathrm{MUT}}\right)$ but reduces to $<2$ years in the absence of such a mutation (IDH wild-type $\left.\left[I D H_{\mathrm{WT}}\right]\right) .{ }^{1}$ Among $I D H_{\mathrm{MUT}} \mathrm{LGG}$, the presence of $1 \mathrm{p} / 19 \mathrm{q}$ chromosomal codeletion ( $I D H_{\mathrm{MUT}}$-Codel) predicts sensitivity to chemoradiation therapy and further improves overall survival

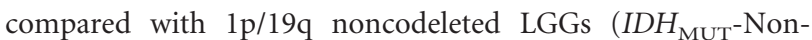
codel). ${ }^{1,2}$ Moreover, the impact of surgery appears to differ among the molecular subtypes. ${ }^{3,4}$ Therefore, noninvasive, presurgical imaging biomarkers that can reliably predict genomic subtypes are of strong clinical interest.

Diffusion-weighted imaging and diffusion tensor imaging are quantitative MR imaging techniques that probe microstructural tissue characteristics by observing the rate and directionality of restricted water diffusion. Both DWI and DTI have demonstrated sensitivity to $I D H$-mutation and $1 \mathrm{p} / 19 \mathrm{q}$ codeletion status in 


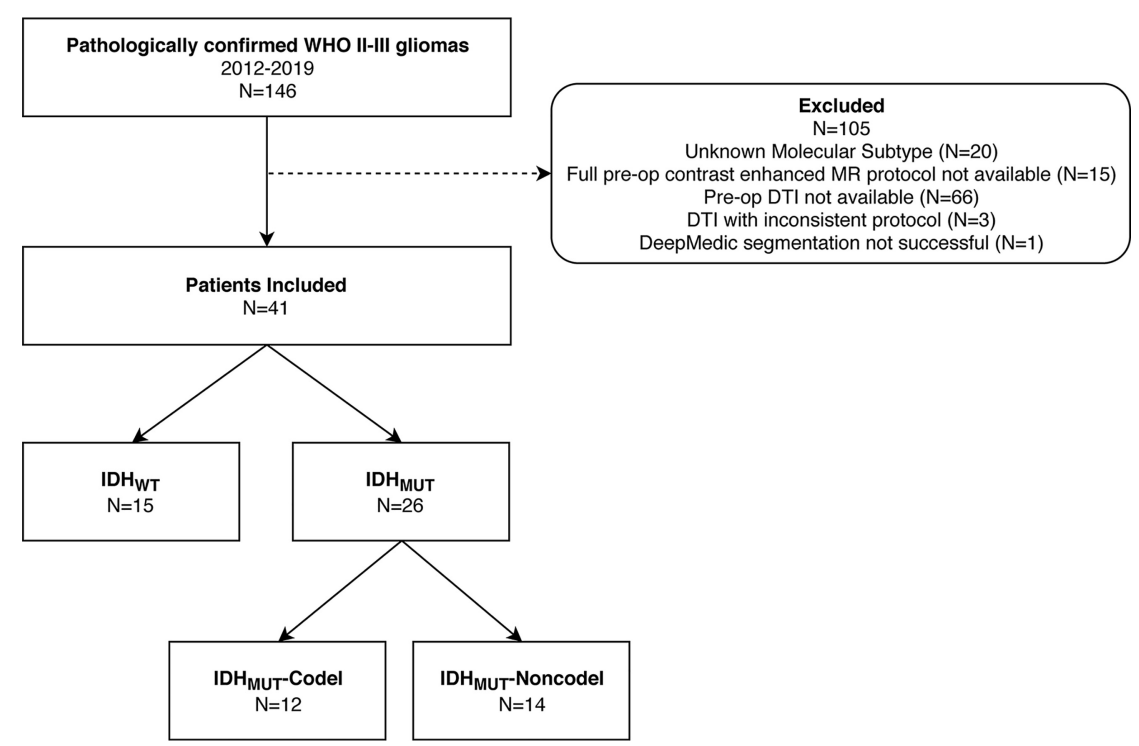

FIG 1. Patient population flow chart.

LGGs via differences in the ADC and/or fractional anisotropy (FA) ${ }^{5-8}$ which have been attributed to differences in cellularity and tumor proliferation rates among subtypes. ${ }^{9}$ While FA has been shown to provide additional value beyond ADC in LGG subtyping, it currently requires the addition of a DTI scan, which is a more specialized imaging technique than DWI and is not part of most routine clinical protocols. Furthermore, because DTI requires acquisitions with diffusion encoding along many directions (at least 6 , typically $\geq 20$ ), its acquisition time is substantially longer than conventional DWI, which only requires diffusion encoding along 3 orthogonal directions. Although MR imaging acceleration techniques such as simultaneous multislice imaging and compressed sensing can greatly accelerate DTI acquisitions, ${ }^{10,11}$ most clinical diffusion $\mathrm{MR}$ imaging scans are still DWIs.

A recently developed DTI reconstruction method called DiffNet (https://github.com/ealiotta/diffnet) uses machine learning to estimate FA from accelerated scans with as few as 3 diffusionencoding directions. ${ }^{12}$ This means that, in principle with DiffNet, the discriminatory power provided by DTI can be extracted from standard DWI scans. While DiffNet has been shown to provide accurate FA map estimates, it remains to be seen whether these estimates add the same value as conventionally reconstructed FA maps from DTI in LGG molecular subtyping.

The purpose of this study was to determine whether FA values estimated using DiffNet from diffusion MR imaging scans with only 3 diffusion-encoding directions add discriminatory value beyond ADC in LGG molecular subtyping. We evaluated the LGG classification in patients who underwent preoperative DTI scans in addition to standard contrast-enhanced MR imaging brain protocols. Classifications were performed using bootstrapped multinomial linear regressions using whole-tumor histogram and texture features from ADC maps alone, ADC plus FA maps, and ADC-plus-DiffNet estimated FA (dFA) maps.

\section{MATERIALS AND METHODS Patient Cohort}

The local institutional review board approved this retrospective study and provided a waiver of the informed consent requirement. One hundred forty-six patients with pathologically confirmed World Health Organization grade II and III gliomas who were imaged at our institution between 2012 and 2019 were identified. Patients with an unknown molecular subtype $(n=20)$, without full preoperative contrast-enhanced MR imaging examinations $(n=15)$, and without preoperative DTI $(n=66)$ or with preoperative DTI with an inconsistent protocol $(n=3)$ were excluded. One additional patient was excluded from analysis due to an unsuccessful tumor autosegmentation (process described below), leaving a total of 41 patients $\left(I D H_{\mathrm{WT}}=15 ; I D H_{\mathrm{MUT}-}\right.$ Codel $=12 ; I D H_{\mathrm{MUT}-}$ Noncodel $=$ 14) who were included in our analysis. This group comprised 26 World Health Organization grade II and 15 World Health Organization grade III tumors and 24 male and 17 female patients with a mean age of 45.9 years (range, $18-76$ years). A flow chart describing the study population is shown in Fig 1.

\section{Neuropathology}

$I D H$-mutation and $1 \mathrm{p} / 19$ codeletion status were tested for all patients in the molecular pathology laboratory at our institution. Formalin-fixed, paraffin-embedded tissue sections were processed routinely for histologic and immunohistochemical analysis. IDH mutation status was tested using immunohistochemistry, in which IDH1 R132H mutations were detected with $\mathrm{H} 09$ antibodies. ${ }^{13}$ In immunohistochemistry cases negative for IDH1 R132H mutations, IDH1/2 mutation status was assessed using DNA pyrosequencing as described previously. ${ }^{14}$ The $1 \mathrm{p} / 19 \mathrm{q}$ codeletion status was determined using dual color fluorescence in situ hybridization ${ }^{15}$ ( $n=38$ ) or chromosomal microarray analysis using the OncoScan (Thermo Fisher Scientific, Waltham, Massachusetts) platform $(n=3)$.

\section{Imaging Protocols}

Patients were imaged on either 1.5T $(n=3)$ or $3 \mathrm{~T}$ scanners $(n=$ 38 ) with pre- and postcontrast $\mathrm{T} 1$-weighted scans $(\mathrm{TE}=1.9-5.0$ $\mathrm{ms}, \mathrm{TR}=9.5-2300 \mathrm{~ms}$, in-plane resolution $=0.5-1.1 \mathrm{~mm}$, slice thickness $=0.9-1.2 \mathrm{~mm})$ as well as T2-weighted $(\mathrm{TE}=82-413$ $\mathrm{ms}, \mathrm{TR}=3200-9000 \mathrm{~ms}$, in-plane resolution $=0.25-1.0 \mathrm{~mm}$, slice thickness $=0.9-5.0 \mathrm{~mm})$ and T2 FLAIR scans $(\mathrm{TE}=80-388$ $\mathrm{ms}, \mathrm{TR}=5000-10,000 \mathrm{~ms}, \mathrm{TI}=2800-2500 \mathrm{~ms}$, in-plane resolution $=0.5-1.0 \mathrm{~mm}$, slice thickness $=0.9-5.0 \mathrm{~mm})$. The DTI protocol included 20 diffusion-encoding directions with $b=1000$ $\mathrm{s} / \mathrm{mm}^{2}$ and $1 b=0$ reference, 1.7 - to $1.9-\mathrm{mm}$ in-plane spatial resolution, 4.0 - to $5.0-\mathrm{mm}$ slice thickness with slice-interleaved single-shot EPI (TE $=6-104 \mathrm{~ms}$ and TR $=3300-4800 \mathrm{~ms}$ ). DTI 
scans were repeated 2-4 times and averaged off-line to improve the SNR.

\section{Tumor Segmentation}

Conventional MR images (T1 pre- and postcontrast, T2, and T2-FLAIR) were coregistered to the reference frame of the T1 postcontrast images. Brain extraction was then performed using the Robust Brain Extraction algorithm (https://www. nitrc.org/projects/robex), ${ }^{16}$ followed by automatic tumor segmentation using DeepMedic (https:/github.com/Kamnitsask/ deepmedic $)^{17}$ as implemented in the Brain Cancer Imaging Phenomics Toolkit (https://captk.projects.nitrc.org/). ${ }^{18}$ Following autosegmentation, tumor volumes were reviewed for obvious errors or segmentation failures, and clearly spurious regions were removed. The DeepMedic output included specific regions for edema, contrast-enhancing gross tumor, and nonenhancing tumor, but all regions were combined into single, whole-tumor ROIs. These ROIs were then registered onto the lower resolution DTI reference frame.

\section{ADC and FA Reconstruction}

ADC and FA values were reconstructed off-line from DTI using conventional linear-least-squares fitting and Eigensytem decomposition ${ }^{19}$ at each voxel using custom Matlab code (MathWorks, Natick, Massachusetts).

Subsets containing only 3 diffusion encoding directions were then extracted from the full DTI scans to replicate conventional DWI acquisitions and simulate a scenario in which DTI was not acquired. These directions were selected to maximize orthogonality and thus closely mimic a DWI acquisition. dFA maps were then computed from these undersampled scans by applying the DiffNet neural network at each voxel. ${ }^{12}$ DiffNet consists of a multilayer perceptron neural network design with 2 hidden layers and a total of 200 nodes and was previously trained to compute FA values from undersampled DTI signals at individual voxels. DiffNet can estimate FA without a full DTI dataset because it bypasses the intermediary tensor-model-fitting reconstruction step and directly infers FA from raw data. DiffNet was implemented in Python (python.org) and is available for download (github.com/ealiotta/diffnet).

\section{ADC and FA Comparisons}

Differences in ADC, FA, and dFA among LGG subgroups were assessed by computing mean whole-tumor values for each parameter and comparing distributions among subgroups using the Student $t$ test. Statistical significance was assessed using the Bonferroni correction for multiple comparisons in which $P$ values $<0.008(.05 / 6)$ were considered statistically significant.

\section{Histogram Feature Extraction}

To assess the full distributions of each DTI parameter, we extracted several histogram features for ADC, FA, and dFA within whole-tumor ROIs. As described elsewhere, ${ }^{6}$ 10th, 25th, 50th, 75th, 90th percentile values, skewness, and kurtosis were computed from ADC, FA, and dFA distributions.

\section{Texture Feature Extraction}

Texture analysis of the ADC, FA, and dFA maps was performed using gray level co-occurrence matrices as previously described in this context. ${ }^{6}$ For each map, homogeneity, correlation, energy, and contrast were computed within the wholetumor ROIs using a Matlab-based radiomic toolbox (https:// github.com/mvallieres/radiomics) developed by Vallières et al. ${ }^{20}$ Gray level co-occurrence parameters were computed within each ROI using equal-probability quantization at 8 quantization levels. The number of radiomic features was kept purposely small to avoid false-positive discoveries, given our cohort size. $^{21}$

\section{Multivariate Classification}

In total, 12 features (mean, 7 histogram features, and 4 texture features) were compiled for each ADC, FA, and dFA map. To classify $I D H$-mutation and $1 \mathrm{p} / 19 \mathrm{q}$ codeletion statuses using all available features, we developed multivariate logistic regression models using imbalance-adjusted bootstrap resampling. ${ }^{20,22}$ Imbalance-adjusted bootstrap resampling permits robust feature selection and generalizable multivariate modeling with a limited dataset by optimizing model parameters on bootstrapped training/testing data subsets while accounting for class imbalances within subsets at each bootstrap repetition.

First, the optimal feature sets were selected from the available histogram and texture features by optimizing classification performance across 50 bootstrapped samples with models containing between 1 and 10 features. With each repetition, patients were randomly divided into training and testing groups with a $60 \% / 40 \%$ split, and class imbalances in each group (ie, a differing number of $I D H_{\mathrm{MUT}}$ versus $I D H_{\mathrm{WT}}$ or $I D H_{\mathrm{MUT}^{-}}$-Codel versus $I D H_{\mathrm{MUT}}$-Noncodel cases) were corrected by repeating instances from the underrepresented class. Regression models were then generated at each iteration using all combinations of available features to optimize prediction accuracy on the training group. The optimal model order (ie, number of features) was then selected by choosing the simplest model (ie, lowest number of features) for which classification area under the curve (AUC) in the testing group reached a maximum or plateau.

This procedure was repeated using $\mathrm{ADC}, \mathrm{ADC}+\mathrm{FA}$, and $\mathrm{ADC}+\mathrm{dFA}$ features for both $I D H$-mutation and $1 \mathrm{p} / 19 \mathrm{q}$ codeletion classifications, generating an optimized feature set for each case. Once optimal feature sets were determined for each classification scheme, a final prediction model was generated by computing model coefficients using imbalance-adjusted bootstrap resampling with 1000 repetitions and taking the average regression coefficients across repetitions. This process ensured that the classification model was not overfitting to any specific subset of patients used for training. Model performance was then quantified in terms of AUC, sensitivity, and specificity in the testing group with each bootstrap repetition, generating a distribution of each parameter for each model. Model performance was evaluated according to the mean and standard error of each metric across repetitions. 

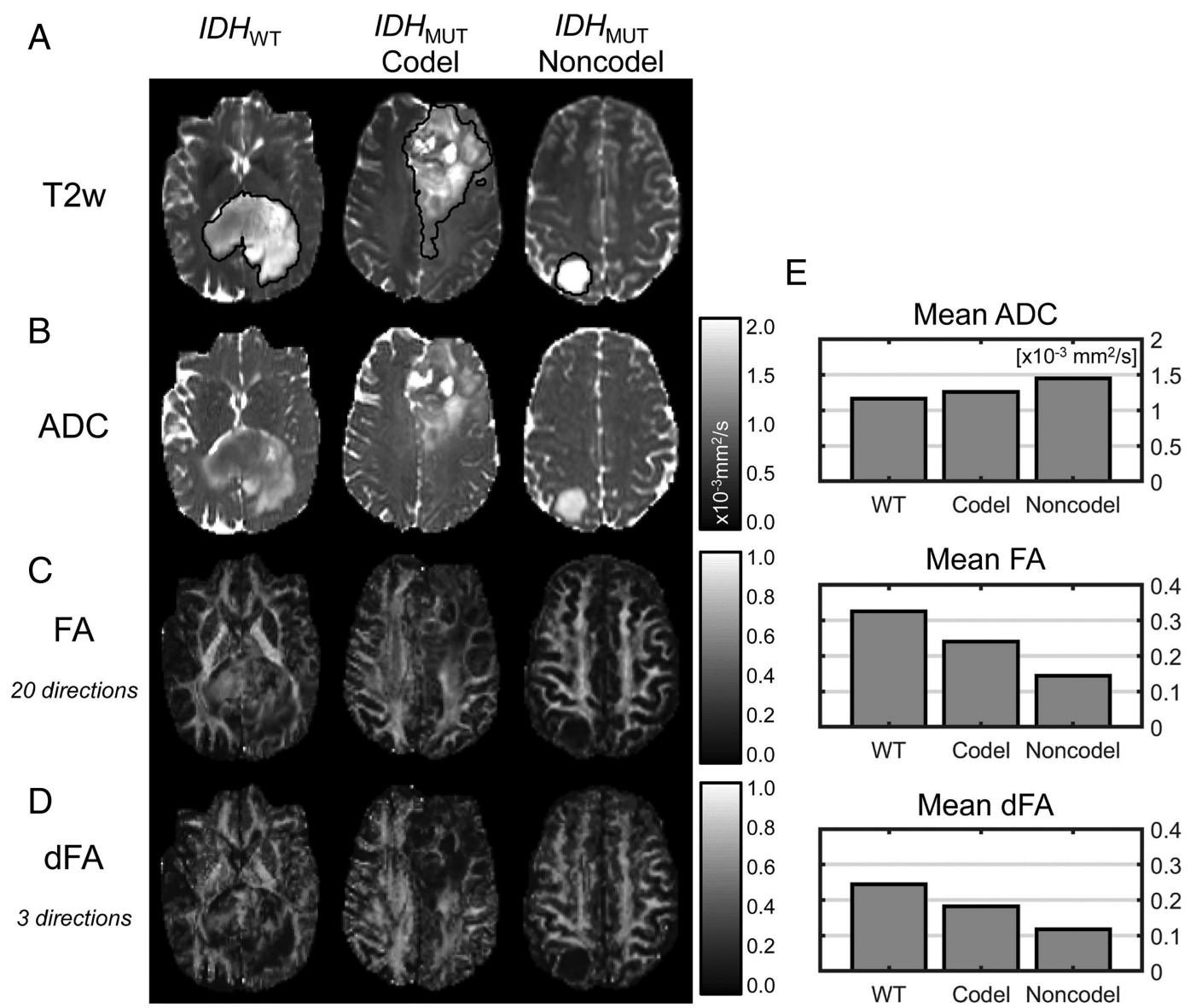

FIG 2. Sample T2-weighted images with tumor segmentations $(A)$, ADC maps $(B)$, FA maps $(C)$, and dFA maps $(D)$ from each LGG molecular subtype as well as mean ADC, FA, and dFA values $(E)$ from these individual cases.
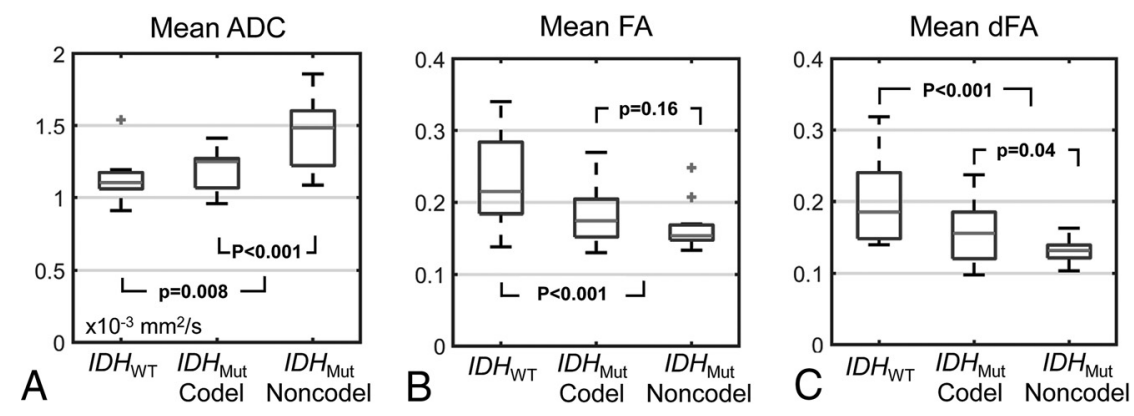

though both were slightly higher in the $I D H_{\mathrm{MUT}^{-}}$-Codel group (FA: $0.18 \pm 0.04$ versus $0.16 \pm 0.03, P=.16$; dFA: $0.16 \pm$ 0.04 versus $0.13 \pm 0.01, P=.04)$. Comparisons among all other histogram and texture features are shown in On-line Tables 1 and 2 .

\section{Classification Feature Selection}

Optimal feature combinations determined in the imbalance-adjusted bootstrap resampling feature selection process are listed in the Table.

Following feature selection, the ADC-only IDH-mutation status classifilines indicate median values, box edges indicate 25 th and 75 th percentiles, and whiskers extend to the full data range, excluding outliers (which are indicated by plus signs). $P$ values $<0.008$ indicate statistically significant differences to account for multiple comparisons.

\section{RESULTS}

\section{Quantitative Subgroup Differences}

Sample ADC, FA, and dFA maps from each LGG subtype are shown in Fig 2, and mean values across patients within each LGG subtype are shown in Fig 3. For $I D H_{\mathrm{WT}}$ versus $I D H_{\mathrm{MUT}}$, significant differences were observed in $\mathrm{ADC}(1.18 \pm 0.16$ versus $1.36 \pm$ $\left.0.24 \times 10^{-3} \mathrm{~mm}^{2} / \mathrm{s}, P=.008\right), \mathrm{FA}(0.22 \pm 0.05$ versus $0.17 \pm 0.04$, $P<.001)$, and dFA $(0.19 \pm 0.05$ versus $0.14 \pm 0.03, P<.001)$. For $I D H_{\mathrm{MUT}}$-Codel versus $I D H_{\mathrm{MUT}}$-Noncodel, a significant difference in $\mathrm{ADC}$ was observed $(1.20 \pm 0.14$ versus $1.48 \pm 0.23 \times$ $\left.10^{-3} \mathrm{~mm}^{2} / \mathrm{s}, P<.001\right)$. Insignificant differences were observed in FA and dFA between $I D H_{\mathrm{MUT}^{-}}$-Codel and $I D H_{\mathrm{MUT}^{-N o n c o d e l}}$, cation included only 1 histogram feature (75th percentile ADC); the ADC + FA model contained 3 histogram features (2 ADC features and 1 FA feature) and 3 texture features (1 ADC feature and 2 FA features); and the ADC + dFA model included 2 histogram features and 2 texture features $(1 \mathrm{ADC}$ and $1 \mathrm{FA}$ feature each).

For $1 \mathrm{p} / 19 \mathrm{q}$ codeletion status classification, the ADC-only model used 1 histogram feature (50th percentile ADC), the ADC + FA model used 5 histogram features (3 ADC features and 2 FA features), and the ADC $+\mathrm{dFA}$ model used 4 histogram features (2 ADC features and $2 \mathrm{dFA}$ features). 
Features selected in the imbalance-adjusted bootstrap resampling process for each classification scheme ${ }^{a}$

\begin{tabular}{|c|c|c|c|c|c|c|}
\hline \multicolumn{7}{|c|}{ Histogram and Texture Features } \\
\hline & \multicolumn{2}{|c|}{ ADC } & \multicolumn{2}{|l|}{$A D C+F A$} & \multicolumn{2}{|c|}{$A D C+d F A$} \\
\hline & Feature & Weight & Feature & Weight & Feature & Weight \\
\hline \multirow{6}{*}{$\begin{array}{l}I D H_{\mathrm{WT}} \text { vs } \\
I D H_{\mathrm{MUT}}\end{array}$} & ADC 75\% & -6.5 & ADC $10 \%$ & 109.4 & ADC $10 \%$ & 40.4 \\
\hline & & & ADC $90 \%$ & -71.4 & ADC homogeneity & 60.2 \\
\hline & & & ADC energy & 4363.0 & dFA energy & -1459.0 \\
\hline & & & FA $75 \%$ & 227.5 & dFA skewness & -6.45 \\
\hline & & & FA contrast & 24.2 & & \\
\hline & & & FA homogeneity & -346.9 & & \\
\hline \multirow{5}{*}{$\begin{array}{l}I^{I D H_{M U T}}{ }^{-C o d e l} \\
\text { vs IDH } \text { MUT }^{-} \\
\text {Noncodel }\end{array}$} & ADC $50 \%$ & -10.7 & ADC $50 \%$ & -266.8 & ADC 75\% & -62.9 \\
\hline & & & ADC $25 \%$ & 306.8 & ADC $10 \%$ & 40.0 \\
\hline & & & ADC skewness & -17.9 & dFA $50 \%$ & 202.9 \\
\hline & & & FA $75 \%$ & 460.9 & dFA skewness & 11.9 \\
\hline & & & FA skewness & -141.5 & & \\
\hline
\end{tabular}

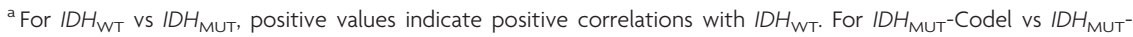

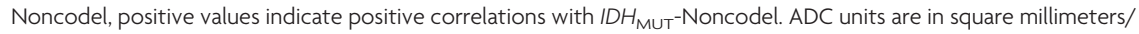
millisecond.

\section{Classification Performance}

For IDH mutation status classification, the ADC-only model achieved AUC $=0.81 \pm 0.03$, sensitivity $=0.84 \pm 0.06$, and specificity $=0.67 \pm 0.05$. The ADC + FA model achieved AUC $=$ $0.90 \pm 0.03$, sensitivity $=0.80 \pm 0.07$, and specificity $=0.80 \pm$ 0.04 . The ADC + dFA model achieved AUC $=0.89 \pm 0.03$, sensitivity $=0.83 \pm 0.06$, and specificity $=0.77 \pm 0.04$.

For codeletion status classification, the ADC only model achieved AUC $=0.83 \pm 0.03$, sensitivity $=0.81 \pm 0.06$, and specificity $=0.73 \pm 0.04$. The ADC + FA model achieved AUC $=$ $0.94 \pm 0.02$, sensitivity $=0.83 \pm 0.05$, and specificity $=0.84 \pm$ 0.05 . The ADC + dFA model achieved AUC $=0.89 \pm 0.03$, sensitivity $=0.76 \pm 0.07$, and specificity $=0.79 \pm 0.05$.

\section{DISCUSSION}

FA estimates obtained from 3-direction diffusion MR imaging scans using DiffNet added discriminatory value in classifying LGGs according to both $I D H$-mutation and $1 \mathrm{p} / 19 \mathrm{q}$ codeletion statuses in addition to ADC maps alone. Although conventional FA values reconstructed from full DTI data provided marginally better classification performance than dFA, both parameters added to the performance of the ADC-only classification. This feature indicates that DiffNet can aid in LGG classification when only DWI is performed. Because DWI scans are included in most standard MR imaging brain protocols, clinical value can be added in many scenarios without the need for additional DTI scans. It should be noted, however, that DTI is often performed to aid in surgical planning and thus is available in many cases.

Several of our findings regarding conventional DTI analysis for LGG classification are consistent with prior work. For example, prior studies have reported lower $\mathrm{ADC}^{5,8,23-25}$ and higher $\mathrm{FA}^{6,7}$ values in $I D H_{\mathrm{WT}}$ LGG compared with $I D H_{\mathrm{MUT}}$ gliomas. Others have also reported significantly higher $\mathrm{ADC}^{7,26}$ and slightly, but nonsignificantly, lower FA values ${ }^{7}$ in $I \mathrm{AH}_{\mathrm{MUT}}{ }^{-N o n-}$ codel versus $I D H_{\mathrm{MUT}}$-Codel tumors.

Other imaging features have previously been used to classify gliomas into molecular subtypes. These include conventional imaging characteristics based on tumor location, ${ }^{27,28}$ contrast enhancement, ${ }^{27,28}$ margins, ${ }^{29,30}$ T2-FLAIR mismatch, ${ }^{31,32}$ and calcification. $^{33}$ Advanced techniques including perfusion MR imaging, ${ }^{5,23,34,35}$ MR imaging spectroscopy, ${ }^{28,36,37}$ and radiomic texture analysis $^{22}$ have also demonstrated discriminatory value. These features can potentially be combined with FA and ADC to further improve classification performance. Additional improvements may also be achievable through more sophisticated classification schemes than the logistic regressions used in this work, such as support-vector machines or random forests.

ADC energy had positive regression coefficients in the IDH-mutation classification, which indicates that uniform ADC values within the tumor are associated with $I D H_{\mathrm{WT}}$. On the other hand, FA homogeneity and dFA energy (both of which indicate parameter uniformity within the tumor) had negative regression coefficients indicating negative associations with $I D H_{\mathrm{WT}}$. These results further indicate that FA and dFA provide complementary information to ADC for LGG classification and agree with prior study results showing higher ADC orderliness and a lack of locally correlated FA values in $I D H_{\text {WT }}$ tumors. ${ }^{6}$

Our finding that increased ADC skewness is associated with $I D H_{\mathrm{MUT}^{-}}$-Noncodel tumors is also consistent with prior work. ${ }^{6}$ However, these authors also found FA energy and correlation as a significant predictor of $I D H_{M U T}$-Noncodel. Neither these nor any ADC, FA, or dFA texture features were selected in our final codeletion-status classification models.

Notably, our codeletion-status classification models included FA skewness and dFA skewness but in opposite directions (increased FA skewness was associated with $I D H_{\mathrm{MUT}^{-}}$-Codel while increased dFA skewness was associated with $I D H_{\text {MUT }}$-Noncodel). This is a surprising finding but may be due to a known FA-dependent bias in dFA. It was shown previously that dFA has a negative bias that gets larger as FA values increase, ${ }^{12}$ which affects the shape of dFA distributions. This can alter distribution skewness and thus associated relationships with molecular subtypes.

This study has limitations that should be discussed. This is a retrospective, single-institution study with a relatively small sample size, and prospective validation on larger samples is necessary. We have made DiffNet publicly available in hopes of facilitating independent validation of our results at separate institutions. Furthermore, although there is no technical difference between a subsampled DTI dataset containing only 3 diffusion-encoding directions and a conventional DWI scan, prospective analysis including only true DWI data is warranted.

\section{CONCLUSIONS}

DiffNet neural network-derived FA estimates based on 3-direction DTI scans improve $I D H$-mutation and $1 \mathrm{p} / 19 \mathrm{q}$ codeletion classification in LGGs compared with ADC values alone. The application of the DiffNet neural network to conventional DWI data may improve the prediction of LGG molecular subtypes. 
Disclosures: Sohil H. Patel—RELATED: Grant: Radiological Society of North America Research Scholar Grant*; UNRELATED: Other: I provided written expert opinion in a malpractice lawsuit.* *Money paid to the institution.

\section{REFERENCES}

1. Brat DJ, Verhaak RG, Aldape KD, et al; Cancer Genome Atlas Research Network. Comprehensive, integrative genomic analysis of diffuse lower-grade gliomas. N Engl J Med 2015;372:2481-98 CrossRef Medline

2. Jenkins RB, Blair H, Ballman KV, et al. A t $(\mathbf{1} ; 19)(\mathbf{q 1 0} ; \mathbf{p} 10)$ mediates the combined deletions of $1 p$ and $19 q$ and predicts a better prognosis of patients with oligodendroglioma. Cancer Res 2006;66:9852-61 CrossRef Medline

3. Wijnenga MM, French PJ, Dubbink HJ, et al. The impact of surgery in molecularly defined low-grade glioma: an integrated clinical, radiological, and molecular analysis. Neuro Oncol 2018;20:103-12 CrossRef Medline

4. Kawaguchi T, Sonoda Y, Shibahara I, et al. Impact of gross total resection in patients with WHO grade III glioma harboring the IDH $1 / 2$ mutation without the $1 \mathrm{p} / 19 \mathrm{q}$ co-deletion. J Neurooncol 2016;129:505-14 CrossRef Medline

5. Leu K, Ott GA, Lai A, et al. Perfusion and diffusion MRI signatures in histologic and genetic subtypes of WHO grade II-III diffuse gliomas. J Neurooncol 2017;134:177-88 CrossRef Medline

6. Park YW, Han K, Ahn SS, et al. Whole-tumor histogram and texture analyses of DTI for evaluation of IDH1-mutation and 1p/19q-codeletion status in World Health Organization grade II gliomas. AJNR Am J Neuroradiol 2018;39:693-98 CrossRef Medline

7. Xiong J, Tan W, Wen J, et al. Combination of diffusion tensor imaging and conventional MRI correlates with isocitrate dehydrogenase $1 / 2$ mutations but not $1 \mathrm{p} / 19 \mathrm{q}$ genotyping in oligodendroglial tumours. Eur Radiol 2016;26:1705-15 CrossRef Medline

8. Suh $\mathrm{CH}$, Kim HS, Jung SC, et al. Imaging prediction of isocitrate dehydrogenase (IDH) mutation in patients with glioma: a systemic review and meta-analysis. Eur Radiol 2019;29:745-58 CrossRef Medline

9. Beppu T, Inoue T, Shibata Y, et al. Fractional anisotropy value by diffusion tensor magnetic resonance imaging as a predictor of cell density and proliferation activity of glioblastomas. Surg Neurol 2005;63:56-61; discussion 61 CrossRef Medline

10. Setsompop K, Gagoski BA, Polimeni JR, et al. Blipped-controlled aliasing in parallel imaging for simultaneous multislice echo planar imaging with reduced g-factor penalty. Magn Reson Med 2012;67: 1210-24 CrossRef Medline

11. Wu Y, Zhu Y, Tang Q, et al. Accelerated MR diffusion tensor imaging using distributed compressed sensing. Magn Reson Med 2014; 71:763-72 CrossRef Medline

12. Aliotta E, Nourzadeh H, Sanders J, et al. Highly accelerated, modelfree diffusion tensor MRI reconstruction using neural networks. Med Phys 2019;46:1581-91 CrossRef Medline

13. Capper D, Weissert S, Balss J, et al. Characterization of $\mathbf{R} 132 \mathbf{H} \mathbf{~ m u -}$ tation-specific IDH1 antibody binding in brain tumors. Brain Pathol 2010;20:245-54 CrossRef Medline

14. Felsberg J, Wolter M, Seul H, et al. Rapid and sensitive assessment of the IDH1 and IDH2 mutation status in cerebral gliomas based on DNA pyrosequencing. Acta Neuropathol 2010;119:501-07 CrossRef Medline

15. Riemenschneider MJ, Jeuken JWM, Wesseling P, et al. Molecular diagnostics of gliomas: state of the art. Acta Neuropathol 2010;120: 567-84 CrossRef Medline

16. Iglesias JE, Liu CY, Thompson P, et al. Robust brain extraction across datasets and comparison with publicly available methods. IEEE Trans Med Imaging 2011;30:1617-34 CrossRef Medline

17. Kamnitsas K, Ledig C, Newcombe VF, et al. Efficient multi-scale 3D CNN with fully connected CRF for accurate brain lesion segmentation. Med Image Anal 2017;36:61-78 CrossRef Medline

18. Rathore S, Bakas S, Pati S, et al. Brain Cancer Imaging Phenomics Toolkit (brain-CaPTk): an interactive platform for quantitative analysis of glioblastoma. In: Crimi A, Bakas S, Kuijf H, et al, eds. Brainlesion: Glioma, Multiple Sclerosis, Stroke and Traumatic Brain Injuries. New York: Springer; 2017;10670:133-45 CrossRef
19. Kingsley PB. Introduction to diffusion tensor imaging mathematics, Part III: tensor calculation, noise, simulations, and optimization. Concepts Magn Reson Part A 2006;28A:155-79 CrossRef

20. Vallières M, Freeman CR, Skamene SR, et al. A radiomics model from joint FDG-PET and MRI texture features for the prediction of lung metastases in soft-tissue sarcomas of the extremities. Phys Med Biol 2015;60:5471-96 CrossRef Medline

21. Chalkidou A, O'Doherty MJ, Marsden PK. False discovery rates in PET and CT studies with texture features: a systematic review. PLoS One 2015;10:e0124165 CrossRef Medline

22. Zhou H, Vallières M, Bai HX, et al. MRI features predict survival and molecular markers in diffuse lower-grade gliomas. Neuro Oncol 2017;19:862-70 CrossRef Medline

23. Xing Z, Yang X, She D, et al. Noninvasive assessment of IDH mutational status in World Health Organization grade II and III astrocytomas using DWI and DSC-PWI combined with conventional MR imaging. AJNR Am J Neuroradiol 2017;38:1138-44 CrossRef Medline

24. Lee S, Choi SH, Ryoo I, et al. Evaluation of the microenvironmental heterogeneity in high-grade gliomas with IDH1/2 gene mutation using histogram analysis of diffusion-weighted imaging and dynamic-susceptibility contrast perfusion imaging. J Neurooncol 2015;121:141-50 CrossRef Medline

25. Wasserman JK, Nicholas G, Yaworski R, et al. Radiological and pathological features associated with IDH1-R132H mutation status and early mortality in newly diagnosed anaplastic astrocytic tumours. PLoS One 2015;10:e0123890 CrossRef Medline

26. Fellah S, Caudal D, De Paula AM, et al. Multimodal MR imaging (diffusion, perfusion, and spectroscopy): is it possible to distinguish oligodendroglial tumor grade and $1 \mathrm{p} / 19 \mathrm{q}$ codeletion in the pretherapeutic diagnosis? AJNR Am J Neuroradiol 2013;34:1326-33 CrossRef Medline

27. Lasocki A, Tsui A, Gaillard F, et al. Reliability of noncontrast-enhancing tumor as a biomarker of IDH1 mutation status in glioblastoma. J Clin Neurosci 2017;39:170-75 CrossRef Medline

28. Nakae S, Murayama K, Sasaki H, et al. Prediction of genetic subgroups in adult supra tentorial gliomas by pre- and intraoperative parameters. J Neurooncol 2017;131:403-12 CrossRef Medline

29. Qi S, Yu L, Li H, et al. Isocitrate dehydrogenase mutation is associated with tumor location and magnetic resonance imaging characteristics in astrocytic neoplasms. Oncol Lett 2014;7:1895-902 CrossRef Medline

30. Delfanti RL, Piccioni DE, Handwerker J, et al. Imaging correlates for the 2016 update on WHO classification of grade II/III gliomas: implications for IDH, 1p/19q and ATRX status. J Neurooncol 2017;135: 601-09 CrossRef Medline

31. Patel SH, Poisson LM, Brat DJ, et al. T2-FLAIR mismatch, an imaging biomarker for IDH and $1 \mathrm{p} / 19 \mathrm{q}$ status in lower-grade gliomas: a TCGA/TCIA project. Clin Cancer Res 2017;23:6078-85 CrossRef Medline

32. Broen MP, Smits M, Wijnenga MM, et al. The T2-FLAIR mismatch sign as an imaging marker for non-enhancing IDH-mutant, 1p/ 19q-intact lower-grade glioma: a validation study. Neuro Oncol 2018;20:1393-99 CrossRef Medline

33. Saito T, Muragaki Y, Maruyama T, et al. Calcification on CT is a simple and valuable preoperative indicator of $1 \mathrm{p} / 19 \mathrm{q}$ loss of heterozygosity in supratentorial brain tumors that are suspected grade II and III gliomas. Brain Tumor Pathol 2016;33:175-82 CrossRef Medline

34. Tan W, Xiong J, Huang W, et al. Noninvasively detecting Isocitrate dehydrogenase 1 gene status in astrocytoma by dynamic susceptibility contrast MRI. J Magn Reson Imaging 2017;45:492-99 CrossRef Medline

35. Yamashita $\mathrm{K}$, Hiwatashi A, Togao $\mathrm{O}$, et al. MR imaging-based analysis of glioblastoma multiforme: estimation of $\mathrm{IDH} 1 \mathrm{mutation}$ status. AJNR Am J Neuroradiol 2016;37:58-65 CrossRef Medline

36. Choi C, Raisanen JM, Ganji SK, et al. Prospective longitudinal analysis of 2-hydroxyglutarate magnetic resonance spectroscopy identifies broad clinical utility for the management of patients with IDH-mutant glioma. J Clin Oncol 2016;34:4030-39 CrossRef Medline

37. Tietze A, Choi C, Mickey B, et al. Noninvasive assessment of isocitrate dehydrogenase mutation status in cerebral gliomas by magnetic resonance spectroscopy in a clinical setting. J Neurosurg 2018; 128:391-98 CrossRef Medline 